Rev Biomed 2004; 15:123- 136.

\title{
Farmacognosia: breve historia de sus orígenes y su relación con las ciencias médicas.
}

\author{
Historia de la Medicina
}

Vieyle Cortez-Gallardo ${ }^{1}$, Juan P. Macedo-Ceja ${ }^{1}$, Mauricio Hernández-Arroyo ${ }^{1}$, Gabriel Arteaga-Aureoles ${ }^{1}$, Diana Espinosa-Galván', Juan F. Rodríguez-Landa ${ }^{1,2}$.

${ }^{1}$ Facultad de Química Farmacéutica Biológica, ${ }^{2}$ Laboratorio de Neurofarmacología, Instituto de Neuroeroetología, Universidad Veracruzana, Xalapa, Veracruz, México.

\section{RESUMEN.}

El ser humano desde su origen ha procurado su bienestar y una gran parte lo ha encontrado en la naturaleza, en muchos casos, asociado con aspectos mágico-religiosos. El estudio científico y el uso adecuado de las sustancias de origen natural con fines terapéuticos ha sido sin duda tan antiguo como la astronomía, la física y la medicina. Actualmente, el estudio sistemático de las drogas naturales es abordado por la farmacognosia. Esta ciencia se enfoca particularmente al estudio de los principios activos de origen vegetal, animal y mineral, así como de los derivados que pudieran tener una aplicación terapéutica, comercial o industrial. En un sentido más amplio la farmacognosia abarca el estudio de la historia, el cultivo, la recolección, preparación, preservación, comercialización, distribución, identificación y evaluación de los componentes químicos de origen natural, la farmacología y el uso tradicional de esos compuestos o sus derivados para mejorar la salud y el bienestar del ser humano. En el presente artículo revisamos de manera somera algunas de las aportaciones históricas de las culturas asiáticas, africanas, europeas y americanas que propiciaron el progreso de la farmacognosia como una ciencia establecida, destacando su importancia en el desarrollo de productos naturales con aplicación en la industria farmacéutica, la farmacología y la medicina, así como su relación con las ciencias médicas.

(Rev Biomed 2004; 15:123- 136)

Palabras clave: farmacognosia, farmacia, farmacología, herbolaria, historia de la medicina, medicina natural, plantas medicinales.

\section{SUMMARY.}

Pharmacognosy: brief history of its origins and their relation with the medical sciences.

Since ancient times, human beings have procured their well-being and have found a great part of it in nature, in many cases associated with mystical aspects.

Solicitud de sobretiros: Dr. Juan Francisco Rodríguez-Landa, A.P. 391, Xalapa, 91 000, Veracruz. México. 


\section{Cortez-Gallardo, JP Macedo-Ceja, M Hernández-Arroyo, G Arteaga-Aureoles, y col.}

The scientific study and the appropriate use of bioactive compounds from natural sources for therapeutic purposes are undoubtedly as ancient as astronomy, physics, and medicine. Nowadays, pharmacognosy has undertaken the systematic study of natural drugs. This science focuses particularly on the study of the secondary metabolites of plants and animals, as well as some mineral compounds that might have a therapeutic, commercial or industrial application. In a wider sense, pharmacognosy includes the study of the history, cultivation, gathering, preparation, preservation, commercialization, distribution, identification, and evaluation of chemical components from natural sources and, in part, of the pharmacology and the traditional use of said compounds or their derivatives to improve the health and welfare of human beings and other animals. In the present article, some historical contributions of Asian, African, European, and American cultures that propitiated the development and establishment of pharmacognosy as an independent science are briefly reviewed. Likewise, we emphasize its importance in the development of natural products that could be applied in industry, pharmaceutics, pharmacology, and medicine.

\section{(Rev Biomed 2004; 15:123- 136)}

Key words: pharmacognosy, pharmacy, pharmacology, phytomedicine, herbalist, history of medicine, natural medicine, medicinal plants.

\section{INTRODUCCIÓN.}

La farmacognosia estudia los principios activos de origen natural que pueden poseer un potencial terapéutico o aplicación en la industria. Por lo tanto, son de importancia en el desarrollo de la industria farmacéutica con repercusiones en las ciencias médicas. Además, los estudios derivados de esta ciencia también tienen relevancia en el progreso de la industria alimenticia, cosmética y textil, entre otras.

La palabra farmacognosia etimológicamente significa "conocimiento de los fármacos". Proviene del griego pharmakon que significa remedio y gnosis que quiere decir conocimiento. El término farmacognosia, como tal, fue utilizado por primera vez en 1815 por Seydler en su publicación titulada Analecta Pharmacognostica. En esta obra definía a la farmacognosia como una ciencia enfocada al estudio del conocimiento de las drogas medicinales. A pesar de esta denominación, en algunos países se le designa de otras maneras. Por ejemplo, a principios del siglo XX el farmacéutico Gómez-Pamo denominaba a la farmacognosia como Materia Médica Vegetal y la describía como parte de la farmacia que estudia las características de las especies naturales, sus partes y su aplicación, así como la recolección, conservación, usos y comercialización. En Francia, por su lado, se le define de manera tradicional como Matière Medicale, mientras que en Alemania se le designa con el término de Biología Farmacéutica. Sin embargo, la denominación generalmente aceptada a nivel mundial para la ciencia encargada del estudio de las sustancias de origen natural con aplicación farmacéutica es la de Farmacognosia. Así, en un sentido más amplio, esta ciencia se encarga de estudiar la historia, el cultivo, la recolección, preparación, preservación, comercialización, distribución, identificación y evaluación de los componentes químicos de origen natural. Adicionalmente, también se encarga del estudio y del uso tradicional de esos compuestos químicos o sus derivados y proporciona los elementos necesarios para determinar su actividad farmacológica y mejorar la salud y el bienestar del ser humano y otros animales. La farmacognosia no sólo se enfoca al estudio de sustancias con efectos terapéuticos per se, sino también de moléculas que sirvan como modelo estructural para la síntesis de nuevos compuestos más potentes; así como de materias primas para los procesos de hemisíntesis y obtener sustancias activas como hormonas esteroides, anestésicos locales y antibióticos; además de enfocarse también a la búsqueda de sustancias naturales que pueden ser aplicadas en la industria en general (figura 1).

En el presente trabajo revisaremos de manera somera algunas de las aportaciones históricas del hombre primitivo y de las culturas más recientes establecidas en Asia, África, Europa y América, que

\section{Revista Biomédica}


Farmacognosia: historia y su relación con las ciencias médicas.

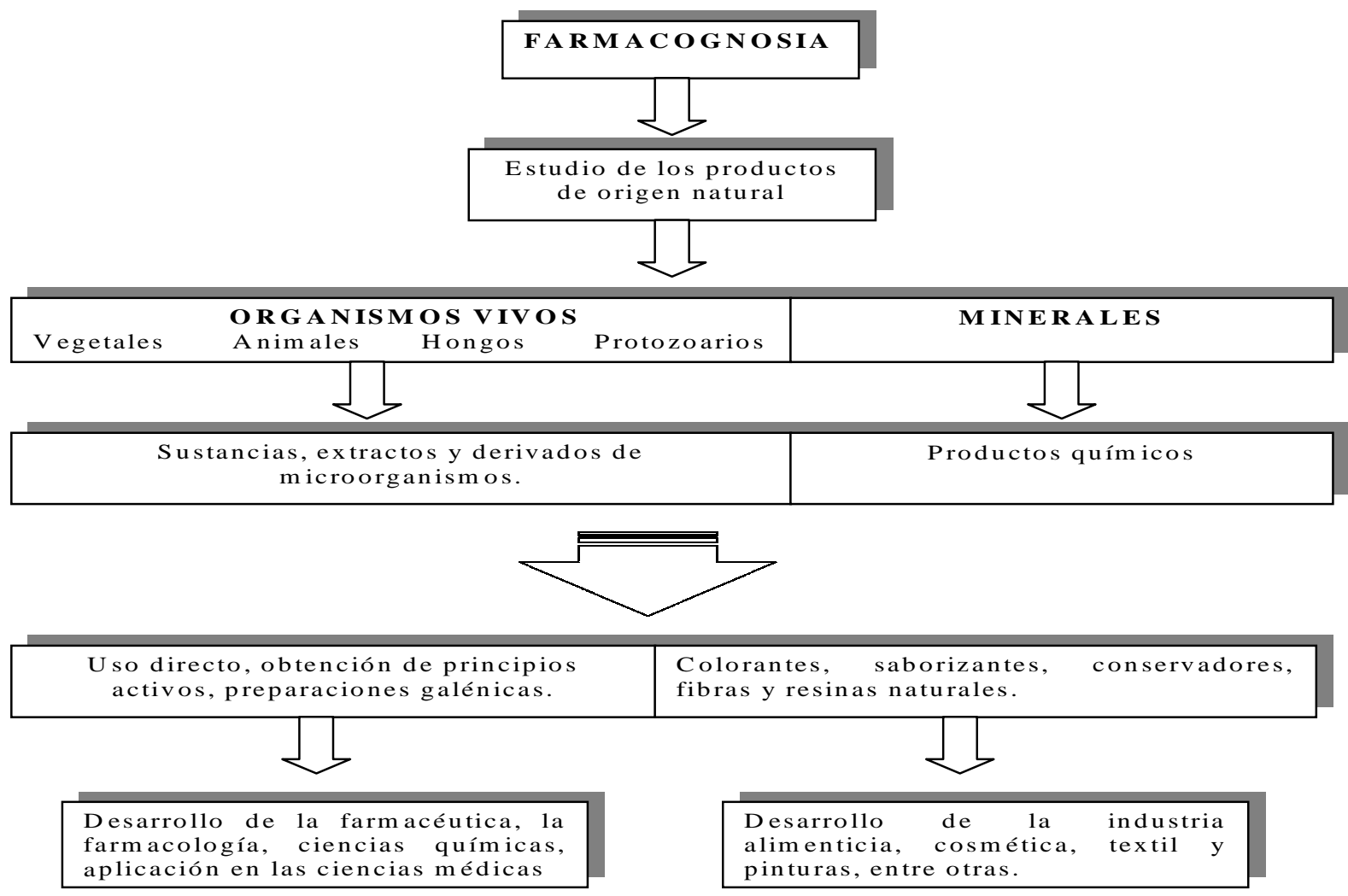

Figura 1.- La farmacognosia estudia los principios activos de origen natural que pueden poseer un potencial terapéutico que repercute en las ciencias médicas, pero también de aquellas sustancias con aplicación en la industria alimenticia, cosmética y textil, entre otras.

propiciaron el desarrollo y establecimiento de la farmacognosia como una ciencia consolidada; asimismo, destacamos su importancia en el desarrollo de productos naturales con aplicación en la industria textil, de alimentos, la cosmética, la farmacéutica, la farmacología y consecuentemente la medicina.

\section{ASPECTOS HISTÓRICOS DE LA FARMACOGNOSIA. \\ LA PREHISTORIA.}

El ser humano desde su origen tuvo que aprender a cazar, vestirse y curarse para buscar la consecución de su bienestar desde una perspectiva "espiritual o científica". Es evidente que esta tendencia, inherente al ser humano, está presente en todo momento y en donde ha existido alguna sociedad, por primitiva que ésta parezca. Estas sociedades practicaban de manera empírica y a base de prueba y error muchas de las disciplinas científicas actuales. Fueron "arquitectos" al construir chozas, "físicos" al dominar el fuego y "médicos" al curar los diversos males que les aquejaban. Este último aspecto llevó al desarrollo de otras disciplinas como la farmacia, la farmacología, la farmacognosia e indudablemente la medicina actual.

El inicio de la historia de la farmacognosia es incierto. No obstante, podemos inferir como debió ser ésta. Si meditamos un poco y pensamos en nuestros ancestros, cazadores y recolectores nómadas, nos daremos cuenta de que para sobrevivir tenían que ser espectadores cuidadosos de la naturaleza. Se vieron en la necesidad de estudiar la conducta de los animales de presa para poder desarrollar estrategias de captura y acercarse a ellos sin que su presencia fuera percibida. Quizás al realizar esta actividad, de manera incidental observaron que algún animal con aspecto "enfermizo" consumía un determinado vegetal - una conducta no común como la de algunos perros al ingerir pasto- y a la postre sanaba. Esta conducta debió ser extraña en los animales de presa y con más razón llamaba la atención de los cazadores que

Vol.15/No. 2/Abril-Junio, 2004 


\section{Cortez-Gallardo, JP Macedo-Ceja, M Hernández-Arroyo, G Arteaga-Aureoles, y col.}

conocían el comportamiento de sus presas. Probablemente, alguno de nuestros antepasados al sentirse enfermo imitó esa conducta del animal y sanó en el mejor de los casos, por medio del ensayo y error - además de su condición de nómada recolector - fue seleccionando vegetales o animales que tenían la propiedad de sanar o mitigar el dolor, pero también de sustancias tóxicas que le podían producir alucinaciones o incluso la muerte. Estas últimas sustancias derivadas de plantas o animales ponzoñosos les fueron sin duda también útiles para la caza de animales o en sus conflictos tribales. Estos fenómenos aparentemente inexplicables - el poder curar o matar - fueron asociados con las nacientes creencias mágicoreligiosas que ya debían estar más o menos desarrolladas para aquellos tiempos (1). Además de los conocimientos sobre medicina que esos pueblos primitivos tenían, también aprendieron a utilizar fibras vegetales para la elaboración de redes para la pesca, cuerdas para arcos y vestido, que combinaron con pieles de animales producto de la caza, lo cual les permitió sobrevivir en aquellos parajes de hostilidad.

Con el paso de los años y el cambio de las tribus nómadas a sedentarias esos conocimientos, entre otros, se consolidaron y el desarrollo de un lenguaje estructurado permitió su difusión con mucha mayor eficacia; en tanto que la jerarquización de labores en las sociedades primitivas permitió que algunos integrantes del grupo comenzaran a especializarse en el manejo y utilización de plantas, animales y minerales con fines curativos. Probablemente, los primeros encargados de esta tarea hayan sido miembros del grupo que se encontraban imposibilitados para el ejercicio de funciones fundamentales como la caza, pero que necesitaban ganar su permanencia y respeto dentro del grupo. Esta tendencia quizás evolucionó y la capacidad de sanar o matar significó poder e influencia dentro del grupo, propiciando la mezcla entre magia, religión y una incipiente ciencia médica que quedó en manos del llamado "chamán” o brujo (1, 2).

Aquel hombre conocedor del uso de los recursos naturales para sanar o matar, a la par de aplicar mezclas de grasas animales, vegetales o minerales, interpretaba "conjuros" que se combinaban con un ambiente rodeado por el humo proveniente de la incineración de plantas y por una decoración mística del lugar de curación que daba un toque sagrado al ritual. Quizás ese "chamán”, conocedor de las propiedades curativas de las plantas, confundió el efecto de las sustancias psicotrópicas con el poder de "seres supremos" que le impidió distinguir entre lo real y lo imaginario.

No obstante, muchos de esos conocimientos antiquísimos que estuvieron envueltos en un ambiente mágico-religioso fueron pasando verbalmente generación tras generación hasta consolidarse como una materia de estudio con la finalidad de mantener el bienestar del ser humano y obtener materias primas naturales que le proporcionaran una mejor calidad de vida. Es aquí donde inicia de manera primitiva el progreso de la farmacognosia hasta llegar a nuestros días como una ciencia consolidada. Así, diferentes civilizaciones - de las llamadas desarrolladas- dieron muestra de un profundo conocimiento del uso y manejo de los recursos naturales para su bienestar, lo cual se puede percibir en culturas de Asia, África, Europa y América, entre otras.

\section{ASIA.}

El continente asiático albergó diversas culturas con características particulares en cuanto al uso de los recursos naturales para su bienestar, los cuales, si bien estuvieron estrechamente relacionados con el aspecto mágico-religioso, también influyeron definitivamente en el desarrollo de la farmacognosia y de las ciencias médicas actuales. Al igual que en otras civilizaciones, en muchos pueblos de Asia siempre estuvo ligada la magia y la religión con los procesos de curación (1), los cuales no se limitaban sólo al uso de plantas sino también de minerales o incluso de animales. En estas culturas al arte de curar se le consideró como sagrado, por lo que era exclusivo de un sector específico de la población, quizás limitando su progreso científico. A pesar de que muchas de las "recetas" y procedimientos descritos por esas civilizaciones son exóticos y carecen de respaldo científico en la actualidad, demostraron que sus autores tenían una gran capacidad de observación de la

\section{Revista Biomédica}




\section{Farmacognosia: historia y su relación con las ciencias médicas.}

naturaleza y del uso del sentido común de manera paralela a las creencias religiosas.

Desde tiempos remotos las culturas establecidas sabían la importancia que tenía el conocer profundamente las propiedades alimenticias, curativas y tóxicas de las plantas y animales que los rodeaban. Los ideogramas sumerios, datados aproximadamente 2500 años a.C., mencionan ya varias "medicinas" de origen vegetal. Los formularios terapéuticos mesopotámicos son conocidos en la actualidad gracias a las tablillas en escritura cuneiforme con listas de drogas cuidadosamente redactadas en tiempos de los sumerios. Estos formularios reunían aproximadamente unas 250 plantas, 120 substancias minerales y 180 remedios de origen animal (algunos de ellos todavía sin identificar) que poseían propiedades medicinales.

Quizás uno de los primeros documentos detallados es el Códice de Hammurabi, rey de Babilonia (1730 - 1685 a.C.), el cual contiene numerosas referencias sobre el uso de plantas curativas que hasta nuestros días siguen siendo utilizadas. La medicina babilónica empleaba sustancias principalmente de origen vegetal. Muestra de ello son los jardines construidos en el reinado de Mardukapalidine II (772-710 a.C.) donde se cultivaban alrededor de 64 especies de plantas con propiedades medicinales, entre las que destacan algunas que siguen siendo útiles hasta nuestros días como el beleño, el eléboro, la mandrágora, el cáñamo y la adormidera, entre otras.

La cultura china tuvo un gran auge en el estudio de las plantas, animales y minerales con fines terapéuticos. Esos conocimientos fueron plasmados en compendios de los cuales el más importante es el llamado Pen tsao kang-mou escrito en el año 2697 a.C durante el reinado del emperador Shen Nung. En él se describen aproximadamente 8160 formulaciones medicinales, algunas de ellas son estudiadas en nuestros días mientras que otras no pudieron evadir el matiz mágico y carecen de un sustento científico. A pesar de esto, los chinos con su gran capacidad de observación aportaron diversos conocimientos enfocados a curar sus enfermedades. Su medicina tradicional era muy variada, lo que se puede apreciar hasta nuestros días. El uso de las hierbas y del té medicinal chino sigue vigente, así como diversas técnicas curativas como la acupuntura, utilizada como tratamiento para diferentes dolencias, controlar el estrés y la obesidad. También aportaron a la medicina el alcanfor, la efedrina, el ginseng y el látex de opio. Este último se usó como remedio para la disentería y en la medicina moderna se utiliza como calmante, somnífero y analgésico, junto con otros alcaloides como la morfina y la papaverina (3).

Por otro lado, la medicina hindú se basó principalmente en el uso de las plantas. Los hindúes tenían un gran progreso en cuanto a cirugía se refiere, por ello muchas de las plantas que utilizaron poseían propiedades anestésicas o narcóticas, como la marihuana (Cannabis sativa). Además, desarrollaron antídotos contra la mordedura de serpientes venenosas y fueron de los primeros en utilizar presentaciones farmacéuticas como ungüentos, infusiones, maceraciones y electuarios (medicamentos con la consistencia de la miel).

Se sabe que las plantas tropicales son las que poseen una mayor cantidad de principios activos. En la cultura hindú se introdujeron muchas plantas de este tipo enfocándose a la prolongación de la vida. Una característica importante es que los hindúes manejaban un sistema de recolección - que aunque poseía un carácter mayormente ceremonial - también tenía datos que se justifican científicamente; por ejemplo, algunas plantas debían recolectarse frescas por la mañana o por la noche, esto se explica debido a que hay plantas que poseen una mayor concentración de principios activos a determinadas horas del día. Asimismo, las colectas no debían realizarse en lugares cercanos a un cementerio o a aguas contaminadas, debido a que podrían originar otras enfermedades por la proliferación de bacterias u otros microorganismos patógenos que no se eliminaban al ser preparadas.

Los árabes, por su lado, fundaron su propia escuela donde estudiaban botánica y química, surgiendo entonces la alquimia gracias a las técnicas avanzadas de laboratorio que ellos mismos establecieron. Los árabes fueron de los primeros en legislar la profesión farmacéutica, de donde derivaron 


\section{Cortez-Gallardo, JP Macedo-Ceja, M Hernández-Arroyo, G Arteaga-Aureoles, y col.}

complejas formulaciones médicas que incluían, entre otras, bebidas fermentadas, jarabes, extractos en alcohol y agua destilada. Lo anterior impulsó, en parte, el desarrollo de la farmacognosia al establecer métodos y técnicas químicas de laboratorio que ayudaron a estudiar las propiedades medicinales de las plantas y animales desde una perspectiva estructurada que posteriormente se convirtió en científica.

\section{ÁFRICA.}

Las grandes aportaciones en medicina y farmacia del continente africano nacen principalmente en el antiguo Egipto, cultura cuyo florecimiento se dio a lo largo del río Nilo y de los desiertos cercanos (1). Gracias a sus vestigios, entre ellos las momias y los papiros, es que podemos percatarnos del papel que jugó esta cultura en el desarrollo de la humanidad.

Así como sucedió en otras culturas, la farmacia y la medicina en el periodo dinástico fueron realmente notables y con un contenido mitológico. Los egipcios tomaron como punto de partida una deidad llamada Dyehuty (Thot) - médico de los dioses - " quien creó las recetas curativas y el que otorga el poder a los médicos para liberar del mal a aquellas personas a quienes Dios ama". Nuevamente, como en otras culturas, la magia y la religión se encontraban estrechamente ligadas a los procedimientos curativos de los egipcios, es decir, en sus diagnósticos y curaciones mezclaban productos naturales y conjuros para que surtiera efecto el tratamiento. Los egipcios fueron de los primeros en dedicarse a estudiar las diversas enfermedades que los aquejaban, por qué sanaban y por qué morían.

Los egipcios clasificaron a aquellas plantas y animales que tenían el poder de curar, enfermar o matar. El uso de plantas, tejidos animales y minerales mezclados en cantidades rigurosas y complejas fueron la base de sus farmacopeas; aunque muchas de esas recetas se centraban más en el plano mágico. Parte de esos conocimientos quedaron plasmados en grandes compendios que en la actualidad conocemos como papiros. Estos escritos, en la mayoría de los casos, llevan el nombre de sus descubridores, del lugar de su descubrimiento o de los museos en los que se encuentran depositados. Hoy en día los papiros constituyen uno de los principales legados de los egipcios y su interpretación es fuente importante de conocimiento sobre su cultura. Son diez papiros encontrados y descifrados en los que se habla de la elaboración de recetas médicas y fórmulas mágicas, otros se refieren a temas específicos como la obstetricia y la ginecología; en tanto que otros describen los conocimientos angiológicos.

Uno de los papiros más famosos es el papiro de Ebers (1550 a. C.) copiado en el año IX de la XVIII dinastía. Este documento, de extensión considerable, y carente de un orden preestablecido forma parte del conocimiento de los egipcios sobre la fisiología humana, la farmacia y la medicina. En él se describe la enfermedad y el remedio adecuado para su tratamiento, así como la composición farmacológica, el tipo de administración y el conjuro para que tal curación se lograra. En el papiro de Ebers se describen más de 1000 recetas que incluyen entre otros productos vegetales, a la cebolla, el hinojo, el ajo, el azafrán, algunos psicoestimulantes como el opio, el café y el cacao, así como sustancias minerales como el plomo, el antimonio y el cobre, que en conjunto eran preparados en soluciones acuosas, oleaginosas, en cerveza o miel, lo que facilitaba su administración. Existen algunas similitudes entre los diversos papiros de los egipcios, por ejemplo el papiro de Hearst contiene prescripciones semejantes a las referidas en el papiro de Ebers. Esto se debe probablemente a que ambos papiros datan de la misma época. No obstante, el papiro de Hearst destaca recetas a base de productos vegetales y animales principalmente para el tratamiento de las fracturas y la elaboración de cosméticos, los cuales quizás eran utilizados también en el ritual de momificación de sus congéneres.

El papiro de Kahun, fechado hacia el año de 1850 a.C., es una muestra más de la gran sabiduría egipcia en el campo de las ciencias médicas. Este papiro describe conocimientos sobre la larga evolución en la rama de la ginecología. Otro papiro, el de Grapow y Deines, menciona cerca de setecientos productos naturales para la preparación de medicamentos y perfumes. Finalmente, el papiro

\section{Revista Biomédica}


Farmacognosia: historia y su relación con las ciencias médicas.

médico de Berlín - del cual se tiene sólo la porción final - contiene la descripción de 204 recetas a base de plantas, animales y minerales utilizadas para diversos males que los aquejaban en aquellas épocas.

Cabe señalar que en la mayoría de los papiros egipcios se separan por primera vez los elementos mágico-religiosos de los conocimientos científicos. Por lo que más tarde esos conocimientos pasarían a manos de otras culturas, de donde el griego Hipócrates posteriormente tomaría algunos de los principios y técnicas que llevó a Europa y que influyeron de manera importante en el desarrollo de la farmacia, la medicina y la farmacognosia.

\section{EUROPA.}

En la antigua Grecia se utilizaban fórmulas mágicas, conjuros y otros procedimientos de los que se creía disponían de algún valor terapéutico sobre aquellas personas que padecían alguna enfermedad; sin embargo, esta civilización ya empleaba el uso de medicamentos naturales para la curación de diversas enfermedades (4). De esta cultura surgieron varios personajes que aportaron grandes conocimientos a la medicina y de manera indirecta también propiciaron el progreso de otras ciencias como la anatomía, la farmacognosia, la farmacia y la farmacología.

Hipócrates (entre el siglo IV y III a. C.), conocido hoy en día como el padre de la medicina moderna, dio las directrices del empleo adecuado de las plantas medicinales y fundó los conceptos básicos de la semiología moderna. Propició el ayuno y la hidroterapia, sentó las bases de lo que hoy conocemos como higiene, así como el valor de una correcta alimentación para tratar los padecimientos que aquejaban a las personas de aquella época bajo su aforismo "Que tu alimento sea tu medicina", lo cual se basaba en la creencia de que el cuerpo en gran parte es autocurativo y por lo tanto sólo necesitaba de una mínima ayuda de drogas y una dieta adecuada para reestablecer la salud. Aristóteles (384-322 a. C.), por otro lado, al igual que Platón (428-347 a. C.), describió las virtudes medicinales y propiedades tóxicas de un gran número de plantas conocidas en ese entonces, pero ninguno de los dos aportó nada nuevo; es decir, se limitaron a plasmar de manera estructurada la información existente y de la cual disponían; lo cual no demerita su trabajo pues sirvió de base para muchos de los estudios posteriores sobre el uso adecuado de los recursos naturales con fines terapéuticos. Asimismo, Teofrasto (372-287 a. de C) realizó grandes estudios en el campo de la botánica, dejando un acerbo bibliográfico acerca de muchas plantas nuevas en Grecia, así como de otros lugares. Su obra es relevante en el desarrollo de la farmacia, la farmacognosia y la medicina, pues no sólo describió los aspectos botánicos de esas plantas sino también los usos terapéuticos que se les daba.

Galeno (129 - 200 d. C.) en su obra "De simplicium medicamentorum temperamentis et facultativus" examinó aproximadamente 473 medicamentos de origen vegetal, mineral y animal. Sus escritos sobre medicina constituyeron la fuente principal del saber de la medicina y hasta hoy en día sus escritos son indiscutibles. Más adelante, Dioscórides (Siglo I d. de C) fue el médico botánico más importante e influyente en su tiempo y quizás uno de los que realizó los más grandes aportes en el campo de la medicina. Dentro de sus obras resalta "De materia médica" la cual destaca la utilización de las plantas en el mantenimiento de la salud, además resulta importante debido a que en ella se encuentran las bases de los grandes herbarios europeos de la Edad Media, en la cual el empleo de las plantas medicinales sufrió un estancamiento y descrédito debido a la "Santa Inquisición" que en su famosa "cacería de brujas" mandó a quemar en la hoguera a curanderos de la época que realizaban "conjuros" durante sus actos de curación. Por lo tanto, solamente en los monasterios se cultivó el arte de curar gracias al trabajo arduo de monjes y sacerdotes que tradujeron del griego y del latín las obras sobre el empleo de las plantas medicinales y cearon grandes huertos y preparados de algunos vegetales en forma de vinos medicinales.

En el caso de los romanos, su medicina se basaba en la agricultura, pues de las plantas que ellos cultivaban se extraían diversos remedios. Lucius Annaesus Séneca (4 a. C. - 65 d. C.) fue uno de los principales romanos que hicieron aportaciones importantes en el 
V Cortez-Gallardo, JP Macedo-Ceja, M Hernández-Arroyo, G Arteaga-Aureoles, y col.

campo de la medicina natural bajo su lema "Los males que sufrimos son curables y si queremos librarnos de ellos, la naturaleza nos ayuda ya que hemos nacido para estar sanos". Por su lado, Aulo Cornelio Celso junto con Plinio escribieron una enciclopedia monumental acerca de la medicina clásica latina titulada "De Artribus", en la que describe los aspectos más importantes de la medicina de los pueblos antiguos basada en el uso de sustancias de origen natural. Otro documento no menos importante es "Remedica", al cual podría considerarse el tratado más importante en el campo de las ciencias médicas dado que divide a la terapéutica en tres partes fundamentales: la dietética, la farmacéutica y la cirugía.

En general, la antigua medicina romana era practicada por los esclavos y ellos mismos eran los encargados de preparar y administrar los medicamentos, aunque también se veían involucradas personas de más alta jerarquía, las cuales en su mayoría eran médicos de Grecia que iban en busca de una mejor fortuna, por tal motivo la medicina romana tenía una gran influencia de la medicina griega (5). Hay que destacar que en la antigua Roma se daba una mayor importancia a la alimentación, a una vida moderada y al ejercicio físico. Los romanos deben gran parte de sus conocimientos sobre medicina terapéutica a los etruscos, los cuales eran grandes conocedores de las virtudes medicinales de las hierbas. En este caso la medicina era practicada por sacerdotes que al igual que los griegos recurrían a la magia, a conjuros e invocaciones y a la adivinación mediante las entrañas de animales, al uso de cartas pronósticas etruscas, así como procesiones religiosas; aunque hay que tomar en cuenta que los conocimientos de terapéutica que tenían los etruscos derivaron en gran medida de la medicina griega. Así, el intercambio de conocimientos entre estas dos culturas propició el progreso de métodos más adecuados para el tratamiento de diversos padecimientos, utilizando recursos animales, vegetales y minerales, ligado en algunos casos con aspectos mágico-religiosos.

No obstante, nuevos conocimientos sobre el uso de las plantas medicinales y otras teorías sobre las ciencias médicas continuaron llegando a Europa a través de los grandes viajeros comerciantes italianos y del Báltico, así como otros introducidos por los árabes, que entraron en contacto con los eruditos europeos en la zona mediterránea, pero especialmente en España. A fines de la Edad Media, se hizo el descubrimiento de América, y los españoles introdujeron una enorme cantidad de nuevos alimentos y medicamentos, enriqueciendo ampliamente los conocimientos médicos y terapéuticos de la Europa de aquella época.

\section{AMÉRICA.}

La herbolaria indígena precolombina jugó un papel importante en el campo de la medicina debido a que el uso de las plantas medicinales y otros productos de origen natural tenía ya una historia de por lo menos 3500 años. Aunque estudios recientes de la paleobotánica han comprobado la existencia de una historia de más de 4000 años de antigüedad detectada en algunos vestigios de tejidos vegetales y polen petrificado encontrados en las excavaciones en zonas arqueológicas de América que sugieren el uso de especies vegetales que aún en la actualidad son perfectamente conocidas y utilizadas en la medicina tradicional.

El descubrimiento del Continente Americano facilitó la fusión de diversas culturas, así como el enriquecimiento de los conocimientos médicos que los colonizadores poseían. Lo anterior permitió el estudio y la explotación de la herbolaria medicinal de los pueblos indígenas por parte de los europeos quienes buscaban primordialmente productos naturales, que hasta fechas anteriores al descubrimiento de América, las obtenían de oriente. Entre esos productos se encontraban las especias y otros productos naturales utilizados en la cocina europea, pero que también eran ampliamente utilizados en la práctica médica. Así, los conocimientos propios de los europeos fueron enormemente reforzados por los chamanes indígenas que les trasmitieron vastos conocimientos respecto al empleo terapéutico de las plantas, animales y minerales del Continente Americano, los cuales eran ya preparados en forma de infusiones, cocimientos, emplastos, ungüentos y aceites, que favorecían la

\section{Revista Biomédica}


Farmacognosia: historia y su relación con las ciencias médicas.

aplicación de los recursos naturales para padecimientos particulares de aquella época $(6,7)$ y aunque no estaba desligado del aspecto mágicoreligioso como la invocación de dioses, ritos mágicos, sacrificios y el exorcismo, favorecieron sin duda el desarrollo de las ciencias médicas $(1,2)$.

Desde antes del siglo XIV, en Mesoamérica se desarrollaron varias culturas como la olmeca, la teotihuacana, la maya, la zapoteca, la purépecha, la tolteca y la azteca, entre otras; mientras que en Sudamérica tuvo un auge considerable la cultura inca. De la cultura que se tiene una mayor información sobre los conocimientos y prácticas médicas es la azteca debido a su predominio en el altiplano mexicano cuando llegaron los conquistadores europeos, la que aprovecharon para su beneficio durante la invasión de Tenochtitlán y el establecimiento de la Nueva España. Este hecho permitió que la mayor parte de los conocimientos herbolarios de los indígenas fueran documentados en códices. De los datos que se tienen de las demás culturas mesoamericanas no dista en lo esencial de los elementos de la cultura azteca, pues era la heredera del cúmulo de conocimientos herbolarios de sus antecesoras (1); sin embargo, cada una de ellas tuvo conocimientos propios debido a los recursos naturales disponibles en su entorno, los cuales no fueron menos importantes.

De los principales legados sobre herbolaria y medicina de las culturas mesoamericanas tenemos aquellos que se realizaron a la llegada de los franciscanos a la Nueva España (1529). Entre ellos destaca Fray Bernardino de Sahagún quien se estableció en el Imperial Colegio de Santa Cruz de Tlatelolco, que fue la primera casa de estudios superiores fundada en el México antiguo (1536). Con respecto a los aztecas, Sahagún fue comisionado en 1557 para recopilar datos acerca de los indígenas, de quienes recaba información en su idioma original, el náhuatl. Su trabajo conocido como Códice Florentino, en náhuatl, y su versión en español como Historia General de las cosas de la Nueva España (8), es un trabajo pionero de la etnografía Azteca en la que describe los nombres y los usos de múltiples plantas medicinales y materiales de origen animal utilizados por los indígenas para el tratamiento de diversas enfermedades. De entre esas plantas destacan también las que poseen efectos psicotrópicos $(6,9$, 10). Por otro lado, Martín de la Cruz, un curandero indígena, describe los métodos curativos conocidos por él, los cuales se basan en el uso de plantas, derivados de animales y algunos minerales. Como resultado, en 1552 aparece un códice en latín llamado Libellus de medicinalibus indorum herbis (11), cuya importancia no sólo radica en el vasto conocimiento herbolario, sino en su contenido artístico al estar ilustrado con dibujos de plantas medicinales a color (mediante pigmentos vegetales y animales), sus nombres en náhuatl, la descripción de sus efectos y su aplicación. La versión en latín fue hecha por Juan Badiano, con cuyo nombre se conoce en la actualidad a dicho códice. Posteriormente, en 1570 Francisco Hernández es comisionado por Felipe II para estudiar la medicina indígena mexicana de donde resulta la obra Historia de las Plantas, donde nuevamente se destaca el amplio conocimiento en el manejo de las plantas medicinales para el tratamiento y prevención de diversas enfermedades. Asimismo, tenemos referencias sobre el empleo de algunas plantas con fines medicinales en obras históricas como la Nueva Crónica y Buen Gobierno (1587) de Felipe de Guzmán Poma de Ayala (12) y los Comentarios Reales de los Incas (1609-1617) de Garcilazo de la Vega (13), en las que se describen los usos y tradiciones que sobre las plantas se tenían; al igual que en Historia Natural y Moral de los Incas de José de Acosta (9).

No menos importantes son los conocimientos en cuanto al uso de plantas y otros recursos terapéuticos empleados en la medicina antigua maya (14), que a pesar de tener tintes místicos (15), tuvieron cierta influencia en el progreso de la medicina mesoamericana. La Península de Yucatán, actualmente dividida en tres entidades federativas (Campeche, Yucatán y Quintana Roo), albergó a la gran Cultura Maya, que al igual que la mayoría de las culturas americanas, tuvo un gran conocimiento en cuanto al uso de plantas, animales y minerales para el tratamiento de diversas enfermedades, lo que no estuvo separado del toque mágico-religioso (2). Entre los mayas los 


\section{Cortez-Gallardo, JP Macedo-Ceja, M Hernández-Arroyo, G Arteaga-Aureoles, y col.}

ritos de curación, además del aspecto mágico, destacaban los procedimientos quirúrgicos, quiroprácticos y, sobretodo, la farmacopea vegetal. Al igual que en otras culturas, las funciones del curandero, hechicero y sacerdote recaían en la misma persona, de tal manera que el rito de curación era múltiple. Algunos de los procedimientos terapéuticos de los mayas fueron registrados en documentos recopilados por los conquistadores, que posteriormente los enviaron a España. Entre esos documentos destaca el conocido como "Relaciones de los Mayas", donde se hace constar que los mayas conocían y usaban múltiples vegetales para curar sus padecimientos, para cicatrizar sus heridas y sobre todo para contrarrestar el veneno de la víbora de cascabel. Además del uso de los recursos vegetales, los mayas aplicaban técnicas como el masaje $(\mathrm{Padz})$ para restaurar los huesos luxados o apretar con fuerza ( Pedz) en el caso de hinchazones. Para tratar otros dolores utilizaban baños calientes mediante el vapor de hierbas preparadas a manera de cocción y en el caso de las fiebres utilizaban baños de agua fría. En cuanto al uso de recursos de origen animal, hacían punciones con espinas de puercoespín (jub) para el tratamiento de las neuralgias, lo cual reducía el dolor. Los colmillos secos de la víbora de cascabel fueron utilizados para realizar sangrías (coh-cán):, se colocaban debajo de la piel de la frente y el canal del colmillo servía para drenar la sangre. En general, a las personas que ejercían el arte de curar bajo estas condiciones se le llamaban dzac-yac, h-men o hax$b a c$, los cuales jugaron un papel importante en la medicina del pueblo maya y sin duda contribuyeron de alguna manera en el desarrollo de la medicina y las ciencias médicas actuales (16), claro está, junto con todas las aportaciones de otras culturas de América y del resto del mundo.

Tomando en consideración los antecedentes antes señalados de las diversas culturas alrededor del mundo, es evidente que la adquisición empírica acerca del uso de los recursos naturales con fines curativos fue puerta de entrada a un gran cúmulo de conocimientos que de manera amplia podemos considerar el punto de partida para el crecimiento de diversas ciencias. Entre ellas, sin duda influyó en el avance de la farmacognosia, la farmacología, la farmacia y, consecuentemente, de la medicina. Así, el desarrollo científico de la farmacognosia tiene repuntes en la búsqueda de alternativas terapéuticas para el tratamiento de enfermedades que a la fecha carecen de un tratamiento adecuado; además de su aplicación en la industria alimenticia y cosmética, de donde radica la importancia de la farmacognosia en la actualidad.

\section{IMPORTANCIA ACTUAL DE LA FARMACOGNOSIA Y COMENTARIOS FINALES.}

La farmacognosia, una vez establecida como ciencia, se enfocó al estudio de las sustancias de origen natural, poniendo especial interés en la identificación, descripción, análisis, comercio y uso terapéutico de las drogas vegetales (17). Debido al auge que han tenido otras ciencias y al avance tecnológico, en la actualidad la farmacognosia posee un alto grado de perfeccionamiento en sus métodos de extracción de principios activos a partir de fuentes naturales, así como en la biosíntesis de sustancias con aplicación terapéutica e industrial. Además, la obtención de plantas transformadas genéticamente y el cultivo in vitro de tejidos vegetales ha permitido identificar y conseguir principios activos con un gran potencial comercial, que de manera natural sería difícil obtener. Más aún, la integración de diferentes ciencias no sólo ha permitido extraer sustancias activas de vegetales, sino también de animales, protistas y algunos hongos, lo cual ha tenido un impacto importante en el progreso de la industria químico-farmacéutica $(18,19)$.

Los estudios farmacognósicos sobre la biosíntesis y la estructura molecular de las drogas naturales permiten sintetizar compuestos análogos con una mayor actividad biológica y potencia terapéutica. Por ejemplo, diversos anestésicos locales como la procaína, la benzocaína y la lidocaína, se sintetizaron tomando como modelo estructural a la cocaína, un alcaloide natural contenido en las hojas de Erythroxylon coca (3). De este modo no es de extrañarse que dentro de cada grupo farmacológico de principios activos utilizados en la terapéutica exista

\section{Revista Biomédica}


Farmacognosia: historia y su relación con las ciencias médicas.

al menos un compuesto prototipo de origen natural.

Los compuestos de origen natural no sólo poseen una importancia farmacéutica per se, sino que su importancia también radica en que pueden funcionar como precursores en la elaboración de los fármacos semisintéticos. Por ejemplo, la progesterona (considerada una hormona sexual femenina) puede obtenerse a partir de la diosgenina, una saponina esteroidea derivada de la dioscina; biosintetizada por varias especies vegetales del genero Dioscorea. En otros casos, los principios activos naturales pueden ser modificados en su estructura molecular básica con la finalidad de obtener compuestos más estables y efectivos, con escasos efectos colaterales y de fácil asimilación por el organismo.

Actualmente, la farmacognosia no sólo se enfoca a la búsqueda de principios activos causantes de una determinada acción terapéutica, sino de sustancias inocuas per se, pero aptas para generar compuestos biológicamente activos cuando se modifica ligeramente su estructura molecular, como sucede con los heterósidos sapónicos al ser transformados a vitamina D, hormonas sexuales o corticosteroides. Cabe señalar que en estos procesos de hemisíntesis se involucra a microorganismos capaces de adicionar grupos funcionales en las moléculas precursoras, reduciendo considerablemente el proceso de biosíntesis, como ocurre con los esteroles, algunas sapogeninas, los cardenólidos y varios subtipos de alcaloides. Un caso particular es el glucocorticoide triamcinolona que se obtiene mediante hidroxilaciones microbianas en las porciones 11 y 16 y por dehidrogenación entre las posiciones 1,2 de su estructura química. Cabe señalar que sin este proceso de biotransformación microbiana la triamcinolona carecería de importancia comercial. Gracias a los avances en los procesos de hemisíntesis a partir de sustancias naturales el ser humano ha podido disponer, en cantidades prácticamente ilimitadas, de una amplia gama de principios activos con actividad farmacológica semejantes a los encontrados de manera natural en algunos vegetales. Aunque, en muchos casos las sustancias de nueva síntesis provocan efectos adversos que no se presentaban en los prototipos de origen natural.

A pesar del progreso de la síntesis orgánica y la hemisíntesis, muchos principios activos de aplicación farmacéutica se siguen obteniendo de fuentes naturales, debido a que en algunos casos los procesos de síntesis son complejos y costosos, por lo que resulta más rentable obtenerlos a partir de vegetales, animales o algunos microorganismos. Un ejemplo de ello ocurre con diversas sustancias ampliamente utilizadas en la práctica médica, como es el caso de la morfina y la codeína que se siguen extrayendo del opio (Papaver somniferum); la quinina y quinidina de varias especies vegetales del género Cinchona; la reserpina y rescinamina de algunas plantas del género Rauwolfia y la L-DOPA que se obtiene de la haba (Viciafaba), mediante procesos más económicos que si se obtuvieran por procesos de síntesis en laboratorio.

De manera general, la farmacognosia a través de la búsqueda y el aislamiento de nuevos principios activos con aplicación terapéutica, proporciona herramientas firmes para el progreso de otras ciencias como la farmacología, donde ha contribuido en gran parte al desarrollo de nuevos y potentes fármacos para el tratamiento de enfermedades que anteriormente carecían de un tratamiento adecuado. Estas investigaciones han adquirido tal magnitud que hoy en día representan un objetivo primordial en la lucha contra enfermedades "de moda" como la depresión, el cáncer y el síndrome de inmunodeficiencia adquirida (SIDA), entre otras, mediante el usos de principios activos obtenidos de sustancias naturales. Por ejemplo, es ampliamente reconocido el efecto antidepresivo de los extractos preparados a partir de la hierba de San Juan (Hypericum perforatum), los cuales tienen un alto contenido del flavonoide hipericina, cuyo efecto farmacológico es semejante al producido por varios antidepresivos sintéticos como la imipramina y la fluoxetina $(20,21)$; sin embargo, aún es necesario realizar estudios adicionales para identificar los efectos colaterales asociados a su consumo o posibles interacciones farmacológicas con otras sustancias (22). En el caso de los estudios dirigidos a encontrar agentes anticancerígenos, se han 


\section{Cortez-Gallardo, JP Macedo-Ceja, M Hernández-Arroyo, G Arteaga-Aureoles, y col.}

enfocado al estudio de plantas con alto contenido de saponinas triterpénicas que son eficaces en el tratamiento de diversas formas de cáncer en animales de experimentación y en el ser humano (23-25). En otro caso, las investigaciones van también dirigidas a explorar la efectividad de extractos obtenidos de plantas con la finalidad de encontrar alternativas para el tratamiento del SIDA, con algunos resultados satisfactorios $(26,27)$. Por otro lado, el uso de sustancias de origen animal también ha tenido repercusiones novedosas en cuanto a la terapéutica. Por mencionar sólo un ejemplo, es conocido que desde tiempos remotos algunas culturas como la egipcia, la mexica y la maya le atribuían propiedades medicinales al cocodrilo. Los estudios científicos actuales han identificado un péptido al que han denominado "cocodrilina" por haberse aislado por primera vez en la sangre del cocodrilo australiano (Crocodylus porosus), resultado que puede ser la base para desarrollar la siguiente generación de antibióticos para el ser humano (28). A pesar de que en algunos estudios clínicos a base de extractos o con principios activos derivados de plantas o animales son alentadores, en muchos de los casos aún faltan estudios complementarios para comprobar o refutar los efectos terapéuticos que se les atribuye y sólo así poder recomendar ampliamente el uso seguro de los fármacos naturales en la terapéutica clínica.

Por último, no debemos olvidar que muchos compuestos obtenidos de plantas y microorganismos, no sólo tienen una aplicación directa en la terapéutica o la industria, sino que muchas de ellas han servido como instrumento de diversas investigaciones que han cambiado el curso de la humanidad. Por ejemplo, el aislamiento de la colchicina a partir del azafrán de otoño (Colchicum autumnales) además de su uso terapéutico en el tratamiento de la artritis gotosa y como antitumoral, permitió amplias investigaciones en el campo de la citogenética y la biología celular debido a que este alcaloide detiene la división celular en vegetales y animales permitiendo ver los cromosomas y estudiar varios procesos durante la replicación celular (29). Otro ejemplo notable es el grupo de las ciclodextrinas. Estas sustancias se aislaron por primera vez a finales del siglo XIX como producto de la degradación del almidón y fue hasta 1904 cuando se les caracterizó como oligosacáridos. Las ciclodextrinas se obtienen por degradación enzimática del almidón a través de la enzima ciclodextringlicosil transferasa producida por microorganismos como el Bacillus macerans y el Bacillus megaterium, entre otros. La importancia de las ciclodextrinas en la industria farmacéutica es relevante debido a que pueden formar compuestos de inclusión con una amplia gama de fármacos, los cuales una vez encapsulados por las ciclodextrinas modifican o potencian sus efectos terapéuticos. Asimismo, estas sustancias son utilizadas para bloquear los efectos colaterales de algunos medicamentos (como la irritación gástrica que muchos antiinflamatorios no esteroidales producen), además de encapsular olores y sabores desagradables en alimentos y productos farmacéuticos, principalmente (30). Por lo anterior, el papel de la farmacognosia va más allá de la búsqueda de sustancias con aplicación terapéutica, dado que algunos productos de origen natural cuya aplicación es relativamente limitada en el campo farmacéutico, son utilizados y altamente cotizados en la elaboración de perfumes, cosméticos, productos de limpieza y bebidas, además de especias y condimentos utilizados en la industria de los alimentos y la repostería.

En resumen, es indiscutible que el vasto cúmulo de conocimientos sobre la explotación de los recursos naturales con fines medicinales, adquiridos empíricamente por el ser humano a lo largo de miles de años, ha favorecido el progreso de diversas áreas de las ciencias médicas. En el caso particular de la investigación propia de la farmacognosia, contribuyó al desarrollo de la terapéutica, la farmacéutica, la farmacología y la medicina, así como al de la industria cosmética y de los alimentos. Aunque, sin suda, su mayor impacto lo ha tenido en el campo de las ciencias médicas y farmacéuticas al identificar y desarrollar nuevos y potentes agentes medicinales. No debemos olvidar que a pesar de los ilimitados campos de la medicina moderna como son la genómica y la farmacogenética (31), las nuevas tendencias del pensamiento científico han vuelto su vista a la

\section{Revista Biomédica}




\section{Farmacognosia: historia y su relación con las ciencias médicas.}

naturaleza en busca de una vida plena. De tal manera que esto favorece que resurja el interés por los productos naturales como fuente de materia prima para mantener la salud y el bienestar del ser humano en un mundo regido por la tecnología. Sin embargo, no debemos olvidar que lo natural no siempre es benéfico para el organismo, sino que depende de la cantidad, los controles de calidad y su procesamiento, lo cual hace necesario incrementar los estudios científicos para identificar o refutar las propiedades que pudieran ejercer en el ser humano a corto y largo plazo. Finalmente, a pesar de la larga historia acumulada a lo largo de miles de años por nuestros antepasados sobre el uso de los productos naturales para mejorar su calidad de vida, el camino aún es largo.

\section{AGRADECIMIENTOS.}

Durante el desarrollo del presente trabajo VC-G, JPMC, MH-A, GA-U y DE-G, recibieron becas escolares por parte de la Universidad Veracruzana. JFR-L recibió beca para estudios de Doctorado por parte del CONACYT (Reg. 124885) y apoyo parcial de la Dirección General de Estudios de Postgrado de la Universidad Nacional Autónoma de México (DGEP-UNAM).

\section{REFERENCIAS.}

1. Pérez-Tamayo R. La medicina de los pueblos primitivos. En: Pérez-Tamayo R, editor. De la magia primitiva a la medicina moderna. México: SEP, Fondo de Cultura Económica, CONACYT; 1997.p. 17-29.

2. Pavía-Ruz N, Ceballos-Quintal JM, Medina-Escobedo C, Ordóñez-Duran A, Ríos-Rodríguez H. Magia, religión y medicina. Rev Biomed 1998; 9:192-8.

3. Brailowsky S. Las sustancias de los sueños: neuropsicofarmacología. México: Fondo de Cultura Económica; 1995.

4. Pérez-Tamayo R. La medicina en Grecia. En: Pérez-Tamayo $\mathrm{R}$, editor. De la magia primitiva a la medicina moderna. México: SEP, Fondo de Cultura Económica, CONACYT; 1997. p. 30-8.

5. Pérez-Tamayo R. La medicina en el imperio Romano (siglos III a.C. a VI d.C). En: Pérez-Tamayo R, editor. De la magia primitiva a la medicina moderna. México: SEP, Fondo de Cultura Económica, CONACYT; 1997. p. 39-51.

6. Lozaya-Legorreta X. Plantas, medicina y poder. Breve historia de la herbolaria mexicana. Los libros del consumidor. México: Pax México; 1994.

7. Lavielle P, Martínez-Murillo C. Las enfermedades hemorrágicas y su tratamiento en la medicina náhuatl. Rev Biomed 1996; 7: 121-4.

8. De Sahagún B. Historia General de las Cosas de la Nueva España. México:Porrúa; 1982.

9. Murillo JR, Tamés R, Arenas C, Díaz JL, Contreras CM. Efecto del extracto de Tila (Tilia mexicana) como hipnótico potencial. En: Contreras CM, Barragán LA, editores. Avances en el mecanismo de acción de fármacos. México: Masson; 1984. p. 157-66.

10. Díaz JL. Accidentados albores de las ciencias fisiológicas mexicanas. Boletín de la Sociedad Mexicana de Ciencias Fisiológicas 1994; 1:4-6.

11. De la Cruz M. Libellus de medicinalibus indorum herbis. Traducción latina de Juan Badiano. Versión española con estudios y comentarios por diversos autores. México: Ediciones del Instituto Mexicano del Seguro Social; 1964.

12. Guzmán Poma de Ayala F. Nueva crónica y buen gobierno. Paris: Edición Facsimilar; 1936.

13. De la Vega G. Comentarios reales de los Incas. Primera parte 1609, segunda parte 1617, Buenos Aires: Emecé Editores; 1944.

14. Urzaiz-Jiménez C. Los recursos terapéuticos empleados en la medicina antigua de Yucatán. Rev Biomed 2002; 13:5968 .

15. Góngora-Biachi RA. Superstición, magia y enfermedad en el Yucatán del siglo XVI según la versión de Pedro Sánchez de Aguilar. Rev Biomed 1998; 9:250-5.

16. Cervera-Andrade A. Breve historia de la cirugía en Yucatán. Rev Biomed 2002; 13:144-51.

17. Villar del Fresno AM, Bermejo P, Carretero ME. Farmacognosia, conceptos generales. En: Villar del Fresno AM, editor. Farmacognosia general. España: Editorial Síntesis; 1999. p. 19-32.

18. Galarza-Vázquez K. El gran desarrollo de la industria químico-farmacéutica (Primera parte). Médico Moderno 2002, 41(1):13-42.

19. Galarza-Vázquez K. El gran desarrollo de la industria 


\section{6}

\section{Cortez-Gallardo, JP Macedo-Ceja, M Hernández-Arroyo, G Arteaga-Aureoles, y col.}

químico-farmacéutica (Última parte). Médico Moderno 2002; 41(2):8-46.

20. Schulz V. Clinical trials with hypericum extracts in patients with depression--results, comparisons, conclusions for therapy with antidepressant drugs. Phytomedicine 2002; 9:468-74.

21. Kasper S, Dienel A. Cluster analysis of symptoms during antidepressant treatment with Hypericum extract in mildly to moderately depressed out-patients. A meta-analysis of data from three randomized, placebo-controlled trials. Psychopharmacology (Berl) 2002; 164:301-8.

22. Rodríguez-Landa JF, Contreras CM. A review of clinical and experimental observations about antidepressant actions and side effects produced by Hypericum perforatum extracts. Phytomedicine 2003; 10:688-99.

23. Plohmann B, Bader G, Hiller K, Franz G. Immunomodulatory and antitumoral effects of triterpenoid saponins. Pharmazie 1997; 52:953-7.

24. Vickers A. Botanical medicines for the treatment of cancer: rationale, overview of current data, and methodological considerations for phase I and II trials. Cancer Invest 2002; 20:1069-79.

25. Tang W, Hemm I, Bertram B. Recent development of antitumoral agents from Chinese herbal medicines; part I. Low molecular compounds. Planta Med 2003; 69:97-108.

26. Matthee G, Wright AD, Konig GM. HIV reverse transcriptase inhibitors of natural origin. Planta Med 1999; 65:493-506.

27. Vermani K, Garg S. Herbal medicines for sexually transmitted diseases and AIDS. J Ethnopharmacol 2002; 80:4966.

28. Cupul-Magaña FG. Cocodrilo: medicina para el alma y el cuerpo. Rev Biomed 2003; 14:45-8.

29. Velasco A. Fármacos antineoplásicos. En: Velasco A, Lorenzo P, Serrano JS, Andres-Trelles F, editores. Farmacología de Velásquez. Madrid:Interamericana-McGraw Hill; 1993. p. 1088-122.

30. Vila J, Blanco J, Anguiano S, Otero F, Fernández J, Torres JJ. Compuestos de inclusión con ciclodextrinas. Parte I: origen, propiedades y aplicaciones biofarmacéuticas de las ciclodextrinas. Revista SEFH 1991; 15:28-35.
31. Pérez-Tamayo R. La medicina moderna. En: Pérez-Tamayo $\mathrm{R}$, editor. De la magia primitiva a la medicina moderna. México: SEP, Fondo de Cultura Económica, CONACYT; 1997. p. 169210.

\section{Revista Biomédica}

